\title{
Perfiles de susceptibilidad de grupos bacterianos aislados de productos cárnicos en Tunja, Boyacá
}

\author{
Daris A Rosas-Leal', Diana P López-Velandia², María I Torres-Caycedo², Maritza Angarita Merchán²
}

\section{RESUMEN}

Introducción. Las bacterias son organismos que se encuentran en diferentes tipos de ambientes que actúan como reservorios, entre estos, los productos de consumo derivados de los animales. Algunas de estas bacterias son capaces de causar enfermedad a los humanos y, a su vez, han evolucionado generando resistencia a antibióticos, lo cual se ha convertido en un problema de salud pública a nivel mundial.

Objetivo. Describir los perfiles de susceptibilidad de grupos bacterianos provenientes de productos cárnicos y derivados, de dos lugares de abasto de Tunja.

Materiales y Métodos. Estudio descriptivo de corte transversal. Se realizó muestreo de productos cárnicos en los expendios de carne y derivados, en un periodo de tres meses, en dos lugares de abasto de la ciudad de Tunja, de los cuales se tomaron diferentes cortes de productos cárnicos para su posterior análisis.

Resultados. A partir de 160 muestras cárnicas recolectadas de 32 puntos de venta, se aislaron 333 cepas bacterianas, encontrando presencia de bacterias Gram negativas y Gram positivas en un $83.2 \%$ y $16.8 \%$ respectivamente. Por otra parte, los perfiles de susceptibilidad antimicrobiano para estas bacterias mostraron sensibilidad del $19,2 \%$ y $0,9 \%$, respectivamente, a los seis antibióticos utilizados para cada grupo en el estudio.

Conclusiones. Se encontró una alta presencia de bacterias procedentes de los aislados de productos cárnicos, que obliga a la mejora de las condiciones de manipulación y expendio de estos productos, dado que, entre los principales riesgos se encuentra la adquisición de cepas resistentes mediante el consumo de alimentos contaminados.

Palabras clave: bacteria; farmacorresistencia; microbiana; inocuidad de los alimentos; enfermedades transmitidas por alimentos.

\footnotetext{
${ }^{1}$ Banco de Sangre Higuera Escalante, Cúcuta, Colombia

2 Universidad de Boyacá. Tunja, Colombia
}

Correspondencia: Maritza Angarita Merchán Dirección: Carrera 2a Este № 64-169, Tunja, Colombia Teléfono: 7450000 ext. 1202.

Correo electrónico: mangarita@uniboyaca.edu.co,gribac@uniboyaca.edu.co

Citar este artículo así:

Rosas Leal DA, López Velandia DP, Torres Caycedo MI, Merchán MA. Perfiles de susceptibilidad de grupos bacterianos aislados de productos cárnicos en Tunja, Boyacá. Revista Investig Salud Univ Boyacá. 2019;6(2): 19-39. doi: https://doi.org/10.24267/23897325.439 


\title{
Susceptibility profiles of bacterial groups of meat products in Tunja, Boyacá
}

\begin{abstract}
Introduction. Bacteria are found in different types of environments that act as reservoirs, among these consumer products derived from animals. Some of these bacteria are able to cause disease to humans and, it in turn, they have devolved generating antibiotics resistance, for that reason has become a public health problem worldwide.
\end{abstract}

Objective. To describe susceptibility profiles of groups bacterium from meat products and derivatives, in two Tunja's market.

Materials and Methods. Descriptive cross-sectional study, they realized sampling meat's products in meat sale zone and by-products in a three-month period in two Tunja's market, which different cuts of meat products were taken, for further analysis.

Results. From 160 meat samples collected from 32 outlets were isolated 333 bacterial strains, it found presence of Gram-negative and Gram-positive bacteria in $83.2 \%$ and $16.8 \%$ respectively. Furthermore, the profiles of antimicrobial susceptibility for these bacteria, it showed sensibility of $19,2 \%$ and $0,9 \%$ respectively to the six antibiotics used for each group in the study.

Conclusions. It found a high presence of isolated bacterium from meat products, what oblige to improve od manipulation conditions and sale of these products, since, among the principal risks are the acquisition of strains resistant by means consume of these food contaminate.

Key words: bacteria, drug resistance, microbial, food safety, foodborne diseases. 


\section{Perfil de susceptibilidade de grupos bacterianos isolados de produtos à base de carne e derivados em Tunja, Boyacá}

\section{RESUMO}

Introdução. As bactérias são microorganismos que se encontram em diferentes ambientes os quais atuam como reservatórios, entre estes, os produtos de consumo derivados de animais. Algumas das bactérias têm a capacidade de causar doenças em humanos, além disso, têm evoluído gerando resistência aos antibióticos, o qual tornou-se um problema de Saúde Pública a nível mundial.

Objetivo. Descrever os perfis de susceptibilidade de grupos bacterianos isolados de produtos à base de carne e derivados, de dois locais de suprimento de Tunja.

Materiais e métodos. Estudo descritivo e transversal. Realizou-se uma amostragem de produtos à base de carne e derivados por um período de três meses, em locais de suprimento da cidade de Tunja, foram coletadas diferentes secções dos produtos para uma análise posterior.

Resultados. De 160 amostras de produtos à base de carne e derivados coletados em 32 pontos de venda, foram isoladas 333 estirpes bacterianas, com a presença de bactérias Gram negativas y Gram positivas em $83.2 \%$ e $16.8 \%$ respetivamente. Por outro lado, os perfis de susceptibilidade aos antimicrobianos mostraram sensibilidade de $19,2 \%$ e 0,9 \% respetivamente, aos seis antibióticos usados para cada grupo no estudo.

Conclusões. Encontrou-se uma elevada presença de bactérias isoladas de produtos à base de carne e derivados, que obriga a melhorar as condições do manuseio e da despesa dos mesmos, dado que, entre os principais riscos encontra-se a aquisição de estirpes resistentes pelo consumo dos alimentos contaminados.

Palavras-chave: bactéria; resistência a drogas; microbiana; segurança alimentar; doenças transmitidas por alimentos. 


\section{INTRODUCCIÓN}

Las bacterias son organismos que se encuentran en diferentes tipos de ambientes, incluidos animales y el hombre. Estos microorganismos son capaces de causar enfermedad en humanos, un ejemplo son las enfermedades transmitidas por alimentos (ETA); se calcula que anualmente hay alrededor del mundo 600 millones de personas con ETA ( 1 de cada 10 personas) y que 420.000 mueren por esta causa (1). La vigilancia sobre la seguridad e inocuidad alimentaria ha ido incrementando con el paso de los años, sin embargo, la verdadera alerta por las ETAs se encuentra en países en vía de desarrollo, abarcando desde la utilización de agua insegura para la limpieza de alimentos, hasta la deficiencia en los procesos de producción alimentaria (como la mala manipulación), la ausencia de la infraestructura necesaria para el almacenamiento de productos alimenticios, y el empleo de normas regulatorias inadecuadas o mal aplicadas que terminan finalmente contribuyendo a un ambiente de alto riesgo (2).

En este sentido, se evidencia un gran problema relacionado con la presencia de bacterias resistentes a antimicrobianos en alimentos, principalmente aquellos originados a partir de productos cárnicos, lo anterior, dado que a algunos animales les suministran suplementos alimenticios también conocidos como antibacterianos promotores de crecimiento (APC) (3). Esta resistencia se transfiere a otros animales y seres humanos, directamente por contacto e indirectamente a través de la cadena alimentaria (4), en la actualidad la farmacorresistencia se encuentra entre las principales emergencias que afectan a las especies microbianas; es así como, el incremento en la presencia de los microorganismos resistentes tiene una marcada repercusión en la salud pública ya que aumenta los índices de morbimortalidad por infecciones (5). Por lo anterior, la Organización Mundial de la Salud (OMS) recomienda, a las industrias que crían animales para el consumo humano, evitar la utilización indiscriminada de antibióticos para estimular su crecimiento y prevenir enfermedades en animales sanos (6).

En Colombia, la situación de la resistencia en patógenos bacterianos aislados de productos cárnicos ha sido reportada; sin embargo, solo actualmente se cuenta con estudios de forma continua que involucran descripciones de prevalencias, caracterización de fenotipos y genotipos, estudios de costos, vigilancia de la resistencia, entre otros (7-9). El estudio de brotes multirresistentes provenientes de productos cárnicos, permite tener un comportamiento epidemiológico que se puede asociar a diferentes factores $y$, a la vez, genera un valor clínico para la toma de decisiones. Por lo tanto, el objetivo fue caracterizar los perfiles de susceptibilidad en cepas bacterianas aislados de productos cárnicos provenientes de dos lugares de abasto de la ciudad de Tunja. 


\section{MATERIALES Y MÉTODOS}

\section{Recolección, traslado y procesamiento de muestras}

Se realizó un estudio descriptivo de corte transversal, se hizo un muestreo a conveniencia a un total 32 puestos de venta de carnes de dos lugares de abasto de la ciudad de Tunja; de cada puesto de venta se tomaron 5 muestras de cortes de diferentes tipos de carnes incluyendo: carne de res, cordero, cerdo y algunas vísceras (corazón, hígado, intestino delgado, intestino grueso) y embutidos (crudos) procesados artesanalmente. Se incluyó un puesto de venta de pescado, obteniendo un total de 160 muestras durante un periodo de tres meses; para el análisis estadístico se utilizó el programa Excel, herramienta office, versión 2016 para establecer los resultados de frecuencias relativas y absolutas.

Cada muestra correspondió a 250 gramos de carne o productos adquiridos por compra, estas fueron colectadas en bolsas individuales marcadas con el tipo de muestra y número de puesto de procedencia; además, se conservaron en cadena de frío hasta su traslado al laboratorio de investigación de la Facultad de Ciencias de la Salud de la Universidad de Boyacá, para su procesamiento.

Se aplicó el procedimiento estándar empleado por Lavilla Lerma L. et al. en el año 2014 (10), para lo cual se preparó la muestra con $5 \mathrm{~g}$ y utilizando fragmentos de diferentes partes; asimismo, se dispusieron $45 \mathrm{ml}$ de solución salina al $0.85 \%$ estéril (10), homogeneizada entre 3 y 5 minutos (Stomacher para mezcla de muestras marca IUL Instruments Digital) hasta obtener una muestra homogénea (dilución inicial); a partir de esta preparación se realizaron las diluciones para los recuentos en placa y aislamiento de la microflora; se inocularon los medios y se procedió la siembra por extensión en superficie, con perlas de vidrio o rastrillo desechable. Posteriormente, se incubaron a $37^{\circ} \mathrm{C}$ durante 24 horas. Los medios de cultivo empleados en el estudio fueron MacConkey (MK), Agar Salmonella/Shigella (SS), Agar Manitol, Agar Plate Count (APC), Agar nutritivo (AN), Agar Eosina Azul de Metileno (EMB) y Agar Sangre marca OXOID ${ }^{\circ}$.

\section{Recuento de colonias totales}

Luego de someter las muestras a procesos de trituración y mezcla con homogeneizador, se prepararon las diluciones seriadas y se inocularon los medios para recuento (APC o AN), luego, se llevaron a incubación (MEMMERT DIGITAL MODELO ULM-800) a $37^{\circ} \mathrm{C}$ por 24 horas. Las lecturas correspondientes se realizaron en contador de colonias tipo Quebec (Marca: INDULAB modelo 007) determinando el recuento de Unidades Formadoras de Colonia (UFC). 


\section{Aislamiento y caracterización fenotípicas de colonias}

Las colonias seleccionadas para la identificación de género y especie, fueron aquellas con mayor UFC en cada uno de los medios de cultivo selectivos/diferenciales para cada muestra y de acuerdo con las características macroscópicas, permitiendo identificaciones presuntivas. Se realizó caracterización fenotípica mediante el empleo del sistema Crystal Gram negativos BBL ${ }^{\circledR}$ y Crystal Gram positivios ${ }^{\circledR}$.

\section{Determinación de los perfiles de susceptibilidad}

La evaluación del perfil de susceptibilidad se realizó mediante la técnica de difusión en disco (Kirby-Bauer) acorde con la guía M100 del Instituto de Normas Clínicas y de Laboratorio (CLSI) versión 2017 (11). Los antibióticos evaluados para Gram negativos fueron Cefepime (CEF), Cefotaxima (CTX), Ceftriaxona (CRO), Imipenem (IMP), Meropenem (MEM), Gentamicina (CN); para Gram positivos se emplearon Cefoxitin (FOX), Amikacina (AMK), Eritromicina (E), Clindamicina (DA), Oxacilina (OXA) y Penicilina (P).

\section{RESULTADOS}

\section{Recuento de colonias totales}

El recuento inferior correspondió a $10 \times 10^{3} \mathrm{UFC} / \mathrm{g}$ de una muestra de carne de vacuno (murillo) y el recuento más alto $40 \times 10^{6} \mathrm{UFC} / \mathrm{g}$ de una muestra de víscera (hígado). Los recuentos de mesófilos aerobios en placa mostraron que, del total de 160 muestras, 28 muestras (17.5\%) presentaron recuentos inferiores o iguales a $10^{3}$, y las 132 muestras restantes $(82.5 \%)$ presentaron recuentos iguales o superiores a $10^{4}$.

\section{Identificación de géneros y especies bacterianas y perfiles de susceptibilidad}

Se aislaron 333 cepas bacterianas, siendo 277 bacterias Gram negativas y 56 Gram positivas (83.2\% y $16.8 \%$ respectivamente) (Gráfico 1 ). El aislamiento bacteriano de mayor frecuencia fue de bacterias Gram negativas de la familia Enterobacteraceae. 
Gráfico 1. Grupos bacterianos aislados de productos cárnicos

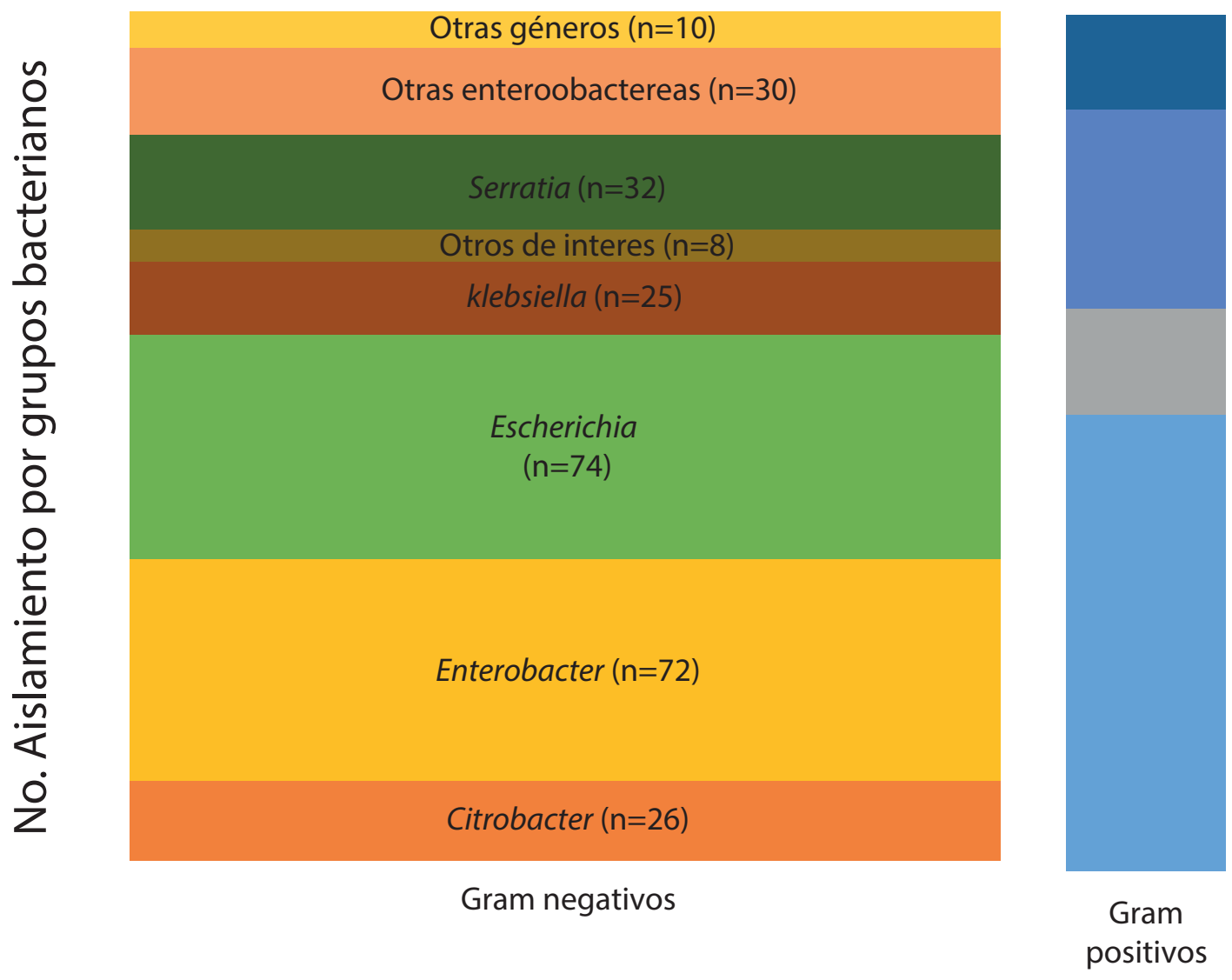

Las bacterias Gram negativas aisladas correspondieron, de acuerdo con el número de aislamientos de mayor a menor, a los géneros fermentadores Escherichia (26.7\%), Enterobacter (26.0\%), Citrobacter $(9.4 \%)$, Klebsiella (9\%), y otras de interés $(2,9 \%)$ por su virulencia que corresponden a géneros fermentadores lentos o no fermentadores como Yersinia (2.2\%), Salmonella (0.36\%) y Shigella
(0.36\%) (Gráfico 2). Dentro de estos géneros, las especies con mayor aislamiento fueron Escherichia coli (26.4\%), seguido de Enterobacter cloacae (8.3\%), Citrobacter freundii (7.9\%), Klebsiella oxytoca (6.1\%), Enterobacter agglomerans (5.4\%) Enterobacter aerogenes (4.3\%), Yersinia enterocolitica (1.1\%) y Yersinia pseudotuberculosis (1.1\%). 
Gráfico 2. Géneros de bacterias Gram negativas aisladas de productos cárnicos.

\section{Bacterias Gram negativas aisladas de productos cárnicos}
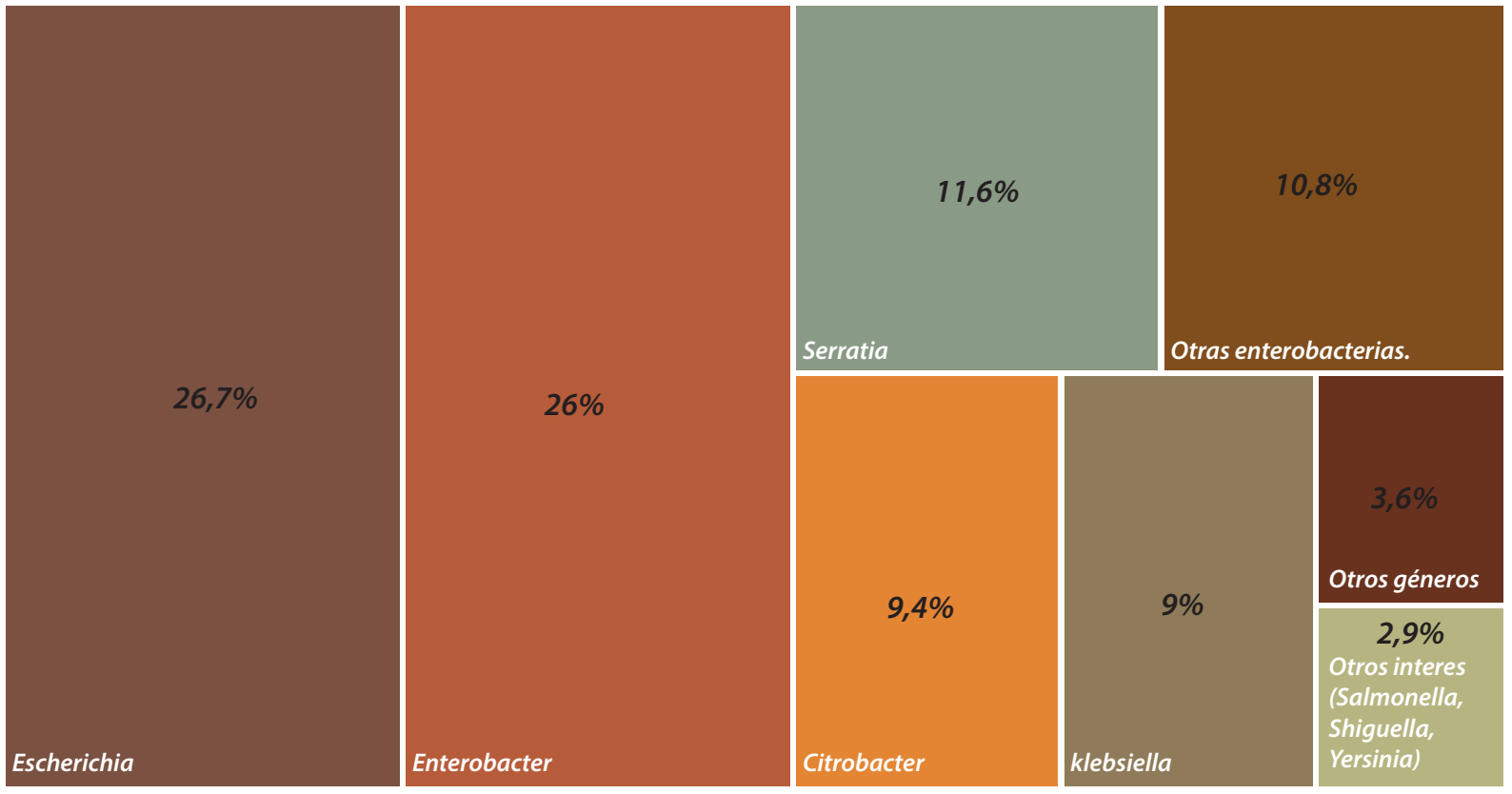

En el grupo de otras enterobacterias aisladas

En relación con las bacterias Gram positivas, de con menor frecuencia se encontraron Serratia liquefaciens (4.7\%), Serratia marcescens (4.0\%), Kluyvera ascorbata (2.50\%), Hafnia alvei (2.2\%), Kluyvera cryocrescens (1.8\%) y Acinetobacter iwoffi y Aeromonas hydrophila (1.1\%). Otros géneros aislados mostraron frecuencias inferiores al $1 \%$ fueron Burkholderia, Chromobacterium, Pantoeba, Plesiomonas, Rahnella, Tatumella y Vibrio (Gráfico 2). los 56 aislamientos se evidencia que el $53.6 \%$ corresponde a especies del género Staphylococcus sp, en donde Staphylococcus aureus (60\%) fue la especie más frecuente dentro de este grupo. Del género Streptococcus sp (23.2\%) la bacteria más aislada corresponde a Streptococcus porcinus (23.1\%). Otros Gram positivos (23,2\%) aislados e identificados fueron Lactococcus (12,5\%), Enterococcus faecalis $(7,1 \%) y$, en menor frecuencia, Bacillus y Micrococcus (1,8\%) (Gráfico 3). 
Gráfico 3. Géneros de bacterias Gram positivas aisladas de productos cárnicos.

\section{Bacterias Gram negativas aisladas de productos cárnicos}

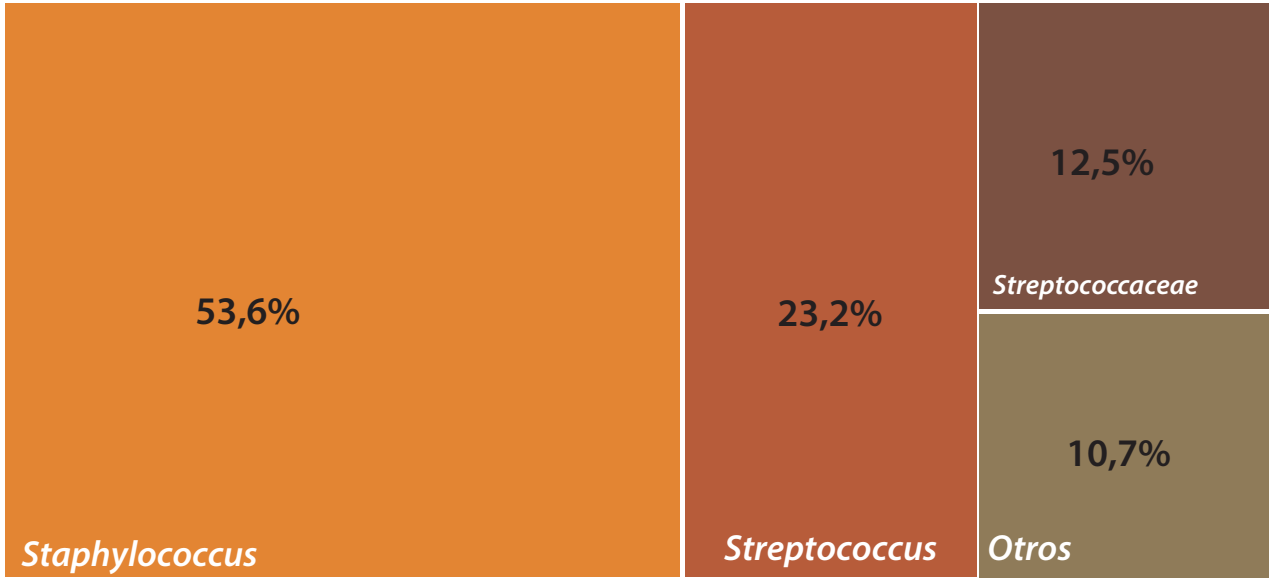

\section{Perfiles de susceptibilidad antibiótica}

De los 333 aislamientos bacterianos, 67 cepas (20.1\%) presentaron sensibilidad a todos los antibióticos probados (Cefepime, Ceftriaxona, Gentamincina, Meropenem e Imipenem); de estos 64 aislamientos (19.2\%) corresponden a bacterias Gram negativas y $3(0.9 \%)$ a Gram positivas, indicando que en los aislamientos de Gram positivas se presenta resistencia bacteriana marcada.

En el grupo de bacterias Gram negativas ( $n=277$ ) se observó sensibilidad para antibióticos como Cefepime (97.8\%), Ceftriaxona (95.7\%), Gentamicina (87\%), Cefotaxima (85.9\%), Meropenem $(64.6 \%)$ e Imipenem (52\%); resultado intermedio de sensibilidad/resistencia se presentó para
Imipenem (39.4\%) y Meropenem (31.8\%). El $18,8 \%$ de las cepas Gram negativas presentaron resistencia a un antibiótico y el $0.4 \%$ a seis.

Por otro lado, las bacterias Gram positivas $(n=56)$, mostraron resultados de resistencia a penicilina (76.8\%), seguido de Amikacina (55.4\%), Clindamicina $(41.1 \%)$, Eritromicina (37.5\%) y Oxacilina (33.9\%); en cuanto a sensibilidad se presentó $64.3 \%$ a Oxacilina, $53.6 \%$ a Clindamicina, $42.9 \%$ a Amikacina, $33.9 \%$ a Eritromicina y $21.4 \%$ a Penicilina; el $44.6 \%$ de las cepas Gram positivas presentaron resistencia a un antibiótico y $28.6 \%$ a cinco, solo el $0.3 \%$ fue sensible a los seis antibióticos utilizados. Los resultados que muestran los perfiles de susceptibilidad - resistencia de los aislados se observan en los gráficos 4 y 5 . 
Gráfico 4. Resultados de antibiogramas de las cepas de bacterias Gram Positivas

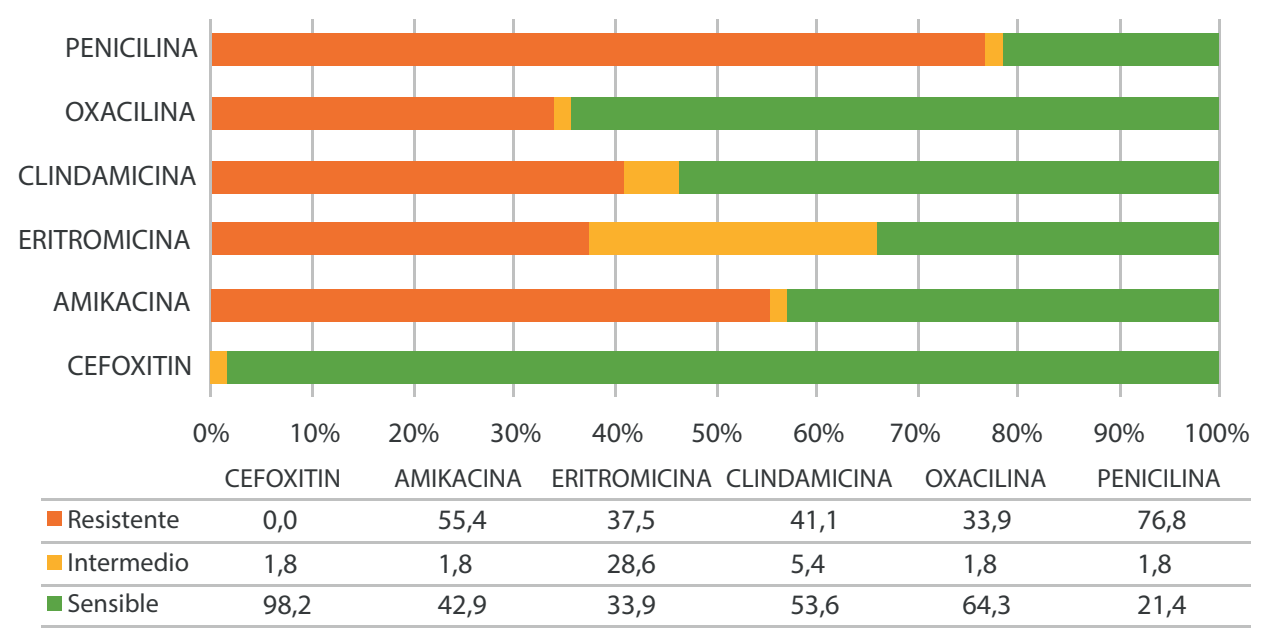

Gráfico 5. Resultados de antibiogramas de las cepas de bacterias Gram negativas

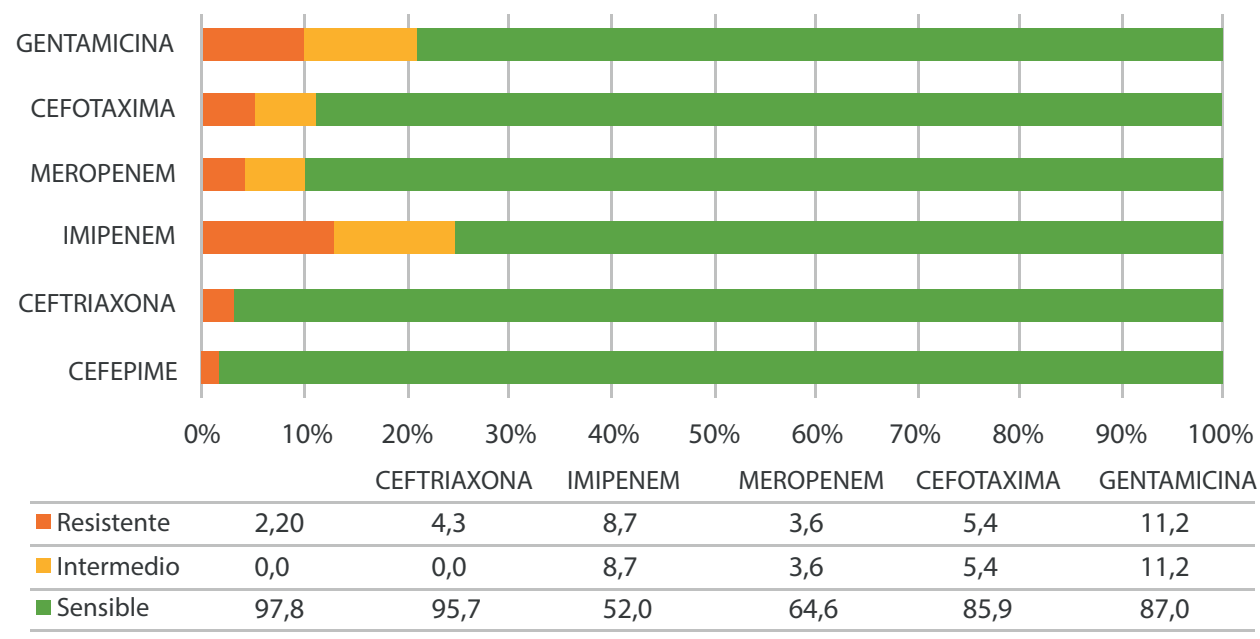




\section{DISCUSIÓN}

De los recuentos bacterianos se evidencia que al ser alimentos crudos y expuestos al ambiente se genera un incremento de la UFC, dado que tienen condiciones de atmósfera gaseosa, temperatura y factores contaminantes, propicios para la colonización de bacterias mesófilas en donde se encuentran grupos bacterianos procedentes del manipulador principalmente. Probablemente, al tratarse de alimentos crudos y expuesto al medio ambiente, se generan recuentos altos de mesófilos, estos pueden ser potencialmente patógenos u oportunistas, además, son una vía de transmisión de resistencia bacteriana. Estos resultados indican le necesidad de mejorar las prácticas de manipulación, dado que se debe asegurar la inocuidad de los alimentos; en consecuencia, es importante resaltar que, de los puestos muestreados en el periodo del estudio, solo uno contaba con refrigerador para mantener la cadena de frío de los productos que allí se expenden. Para los productos cárnicos crudos se exige la cadena de frío entre otras consideraciones (29), por lo tanto, esta es una prioridad para los lugares de abasto de este tipo de productos.

El aislamiento bacteriano de mayor frecuencia fue de bacterias Gram negativas de la familia Enterobacteraceae, las cuales se encuentran relacionados con la flora de humanos y animales; así mismo, se muestra aislamiento de grupos bacterianos provenientes de medio ambiente como agua, suelos y vegetación; como Hafnia alvei, Kluyvera cryocrescens y Aeromonas hydrophila. Otros géneros aislados con frecuencia baja, como Burkholderia, Chromobacterium, Pantoeba, Plesiomonas, Rahnella, Tatumella y Vibrio, han sido reportados en aislamientos de alimentos cárnicos, aguas y suelos, y se han relacionado con casos clínicos causados por patógenos de baja prevalencia $(30,31)$.

Los resultados de identificación fenotípica de bacterias Gram negativas son similares a los de un estudio realizado en Alemania, en el cual, de un total de 500 muestras de pollo y 500 de carne de cerdo, fueron positivas para Escherichia coli $86 \%$ y $49 \%$, respectivamente; así mismo, se identificó una prevalencia de $22 \%$ y $36 \%$ de Enterobacter spp., y en menor proporción, se encontraron otros géneros de enterobacterias como Citrobacter spp y Klebsiella spp (32). Por otro lado, en España, a partir de un muestreo de 50 tiendas, se encontró una alta prevalencia de cepas de Escherichia coli y Serratia fonticola (82 y $16 \%$ respectivamente). Las demás cepas identificadas correspondieron a Proteus vulgaris, Citrobacter koseri, Klebsiella pneumoniae, Enterobacter cloacae, Proteus penneri, Proteus mirabilis, Hafnia alvei y Rahnella aquatilis (33); por otra parte, en Egipto, de un total de 100 muestras de carne cruda (50 de pollo y 50 de carne de res), obtuvieron un total de 228 aislados bacterianos en donde la mayor prevalencia fue de Proteus spp. (60\%), 
seguido de Escherichia coli (38.7\%), Klebsiella spp. (17.3\%) y Citrobacter spp. (13.3\%) (34). En América también se han realizado estudios similares; en México, de un total de 90 muestras se obtuvieron aislamientos de cepas de Escherichia coli (20\%) (35).

Una gran cantidad de la población proveniente de países de bajos y medianos ingresos compra carne de mercados informales, ya que estos productos tienden a ser más económicos y suelen estar localizados cerca de comunidades rurales $(19,20)$, sin embargo, la ausencia de normas de seguridad e higiene en la manipulación y conservación de productos cárnicos, sumado a su composición, favorece el crecimiento de microbioma en niveles no aptos para su consumo. Por lo anterior, este tipo de productos contaminados representa un alto riesgo en cuanto a ETAs a nivel global, especialmente en países en desarrollo, donde los sistemas de gestión de la higiene son implementados de manera deficiente (21-23).

Dentro de los principales patógenos intestinales transmitidos por alimentos se encuentran Salmonella sp., Escherichia coli y Campylobacter sp., entre otras enterobacterias presentes en el tracto gastrointestinal de los animales y las cuáles son causantes de contaminación cruzada, resultado de una técnica de sacrificio y normas de higiene deficientes, desde las plantas de sacrificio (24-26).
En el contexto nacional se han realizado varios estudios, entre ellos, uno llevado a cabo en la ciudad de Cartagena, donde se identificaron 36 muestras con Escherichia coli en niveles no aceptables, según las normas del Instituto Nacional de Vigilancia y Medicamentos y Alimentos (INVIMA) (36). Otro estudio realizado en Bogotá, en el cual se analizaron 38 muestras cárnicas, arrojó que 16 de ellas fueron positivas para Escherichia coli (57.1\%) (37); de igual forma, en Pamplona (Norte de Santander) se identificó Escherichia coli como indicador de contaminación fecal a partir 51 muestras de pescado fresco, observándose que en el $8 \%$ de las muestras se presentaban niveles inaceptables para su consumo, según lo establecido por la Comisión Internacional de Especificaciones Microbiológicas (ICMSF) (38).

En cuanto a bacterias Gram positivas, los Staphyloccocus son un género bacteriano frecuentemente estudiado (17) en cárnicos y productos derivados crudos, dada la relación con toxicoinfección alimentaria, otras especies identificadas dentro del estudio como sciuri, vitulus, simulans se han encontrado en agua, vegetales o como etiología de mastitis en rumiantes pequeños(18). Los resultados encontrados se pueden relacionar con diferentes estudios realizados a nivel mundial, en Egipto y Arabia Saudita, se identificó la presencia de Staphylococcus aureus en 250 muestras de carne de diferentes cortes, recolectadas de diferentes supermercados del área 
de estudio, (39). En Estados Unidos, a partir de un muestreo de 3290 cortes de carne, se reportó que 913 muestras de carne fueron positivas para Staphylococcus aureus (40); de igual manera, en India se analizaron un total de 65 muestras de carne cruda (pollo y cabra) de las cuales 30 muestras fueron positivas para Staphylococcus aureus con una prevalencia de $48.57 \%$ en pollo y $43.33 \%$ en cabra (41).

En diferentes países de América se han realizado estudios similares, por ejemplo, en Brasil se identificó la prevalencia y diversidad de las especies de Staphylococcus spp. aisladas de la carne de pollo, siendo las más frecuentes Staphylococcus aureus, Staphylococcus hyicus, Staphylococcus intermedius, Staphylococcus epidermidis, Staphylococcus lugdunensis, Staphylococcus haemolyticus, Staphylococcus hominus, Staphylococcus lentus, Staphylococcus schleiferi y Staphylococcus cohnii (42).

Entre los hallazgos encontrados para perfiles de susceptibilidad antimicrobiana en este estudio, se evidenció que las 277 cepas bacterianas Gram negativas mostraron patrones similares de sensibilidad para cefalosporinas y aminoglucósidos; sin embargo, también se evidenciaron porcentajes de susceptibilidad intermedia del $8.7 \%$ para Imipenem y 3.6\% para Meropenem, siendo estos dos últimos de gran importancia, ya que son antibióticos utilizados como última opción para tratamiento de infecciones por ser antibióticos de amplio espectro (43), que en un futuro podrían empezar a generar resistencia.

En cuanto al perfil de susceptibilidad antimicrobiana en bacterias Gram positivas, se encontró que manejaron patrones de resistencia mayores. Diversas investigaciones en el ámbito mundial describen comportamientos diferentes sobre los patrones de susceptibilidad antimicrobiana que pueden presentar las bacterias aisladas e identificadas en la presente investigación, así, en Suiza para la familia Enterobacteriaceae, se describió una prevalencia mayor al $50 \%$ de resistencia a antimicrobianos tipo aminoglucósidos, cefalosporinas, fluoroquinolonas, penicilinas, sulfonamidas y tetraciclinas en cepas aisladas a partir de carne (44). Otro estudio realizado en China demostró que el $94 \%$ de los microorganismos aislados de cortes de pollos, patos, cerdos y vacas presentaron resistencia al menos a un fármaco y $83 \%$ de resistencia al menos a tres clases diferentes de antimicrobianos (45); estos resultados difieren de los obtenidos dentro del estudio. Sin embargo, una investigación realizada en Alemania mostró una baja prevalencia de Escherichia coli resistente en carne de caza empaquetada, en comparación con los estudios sobre carne convencional (46), siendo similar a los resultados de este estudio.

De otra parte, en relación con el perfil de susceptibilidad en Gram positivos, un estudio realizado en Suiza demostró que géneros como Staphylococcus 
y Enterococcus aislados de fuentes cárnicas, pescados y mariscos, presentaron resistencia contra glicoproteínas, lincosamidas, macrólidos y nitrofuranos (44). Otro estudio realizado en Chile obtuvo resultados diferentes dados por un bajo porcentaje de cepas que mostraron resistencia a Oxacilina y Cefoxitin (47); en Corea, aislados de Staphylococcus aureus fueron resistentes a penicilinas $(51.2 \%)$, tetraciclina (38.8\%) y ciprofloxacina (33.9\%) (48).

Las bacterias como indicadoras de la manipulación de los alimentos no solo se consideran como indicadores de riesgo de infecciones, tóxico infecciosas incluidas en el grupo de ETAs, sino que actualmente generan alerta por la posible vehiculización de bacterias resistentes en alimentos, que se encuentran en condiciones de producción y comercialización con bajos estándares de calidad relacionados con la seguridad e inocuidad alimentaria; por lo anterior, es determinante ampliar el estudio de las estrategias de buenas prácticas que incluyen la exigencia en indicadores microbiológicos y detección de sustancias derivadas del uso en animales de producción, para así identificar y hacer análisis de riesgo buscando la contención de esta resistencia y la inocuidad (49-51).

\section{CONCLUSIONES}

Los resultados relacionados con los recuentos, grupos bacterianos identificados y sus perfiles frente a los antibióticos, muestran un ambiente que proporciona condiciones para la colonización y generación de reservorios de las bacterias: los expendios (puestos) muestreados pueden optimizar sus condiciones para garantizar mejores prácticas de manipulación, minimización de riesgo de trasmisión de cepas virulentas y resistentes, control del crecimiento bacteriano con métodos de conservación y expendio (como conservación en cadena de frío), mejoramiento locativo y de superficies, utilización de material exclusivo para el manejo de cárnicos y otros procedentes de producción animal, dispensación de los cortes de carne y derivados de manera separada; entre otras prácticas, además de la concienciación de los manipuladores frente a la inocuidad alimentaria y su seguridad, que revierte en estrategias para contrarrestar no solo las ETAS, sino que también contribuye a la multiplicación de clones bacterianos resistentes circulantes.

La identificación de perfiles de resistencia a antibióticos en las cepas aisladas indica uno de los principales riesgos para transmisión de cepas resistentes, que puede llegar a generar consecuencias para la salud humana mediante el consumo de alimentos contaminados por bacterias resistentes, ya sea por consumo del alimento crudo o con baja cocción o por contaminación cruzada del alimento que actúa como reservorio. 
Los problemas sanitarios encontrados a nivel mundial en los alimentos reflejan un alto riesgo de contraer infecciones alimentarias, por lo tanto, las soluciones de estos problemas requieren incrementar la educación sanitaria de los manipuladores y consumidores, así como realizar más estudios donde se determine la calidad microbiológica de estos productos crudos e informar a la comunidad y entes de control para crear medidas con el fin de disminuir este problema.

En relación con la susceptibilidad antibiótica de aislamientos de alimentos como ambiente colonizado por bacterias provenientes principalmente de la manipulación, se debe evaluar la presencia de géneros que expresan resistencia a uno o más antibióticos, lo que supone activar mejores estrategias de manipulación en toda la cadena hasta el consumo, es decir, lo que involucra un sistema de vigilancia y educación permanente, tanto a expendedores como consumidores. Esta resistencia se observa principalmente en los aislamientos de bacterias Gram positivas como Staphylococcus, género en el que se han reconocido fenotipos de resistencia específicos; de otra parte, es necesario generar estudios continuos sobre uso de antibióticos en medicina veterinaria y producción pecuaria, toda vez que esta es una práctica frecuente y podría condicionar un ambiente de presión que genera respuesta evolutiva de géneros bacterianos ambientales, expuestos a la actividad antibiótica.

\section{CONSIDERACIONES ÉTICAS}

El proyecto fue avalado formalmente por el Comité de Bioética de la Universidad de Boyacá (CB 385 del 8 de marzo de 2018), a partir de los establecido en la Resolución 008430 de 1993.

\section{CONFLICTOS DE INTERESES}

Los autores declaran no tener conflicto de interés.

\section{FINANCIACIÓN}

Este proyecto contó con el aporte de la Universidad de Boyacá.

\section{AGRADECIMIENTOS}

A la Universidad de Boyacá, al equipo de trabajo de este estudio y demás personas que intervinieron en su realización.

\section{REFERENCIAS}

1. WHO: World Health Organization. [Internet]. Ginebra:Suiza; [31 octubre de 2017; citado 30 enero 2019]. Inocuidad de los alimentos. [aprox. 2 pantallas]. Available from: https:// www.who.int/mediacentre/factsheets/fs399/es/

2. Jayasena DD, Kim HJ, Yong HI, Park S, Kim $\mathrm{K}$, Choe W, et al. Flexible thin-layer dielectric 
barrier discharge plasma treatment of pork butt and beef loin: Effects on pathogen inactivation and meat-quality attributes. Food Microbiol. 2015;46:51-7. https://doi.org/10.1016/j.fm.2014.07.009

3. CDC: Centers for Disease Control and Prevention. [Internet]. Druid Hills: Estados Unidos; [26 noviembre de 2018; citado 30 enero 2019]. Antibiotic Resistance Threats in the United States. [aprox. 3 pantallas]. Available from: https://www.cdc.gov/drugresistance/ threat-report-2013/index.html

4. Van Boeckel TP, Brower C, Gilbert M, Grenfell $\mathrm{BT}$, Levin $\mathrm{S}$ a, Robinson TP, et al. Global trends in antimicrobial use in food animals. Proc Natl Acad Sci [Internet]. 2015;112(18):5649-54. https://doi.org/10.1073/pnas.1503141112

5. Puig Peña Y, Espino Hernández M, Leyva CastiIlo V. Resistencia antimicrobiana en Salmonella y E. coli aisladas de alimentos: revisión de la literatura. Panor Cuba y Salud. 2011;6(1):30-8.

6. WHO: World Health Organization. [Internet]. Ginebra:Suiza; [07 noviembre de 2017; citado 30 enero 2019]. Dejemos de administrar antibióticos a animales sanos. [aprox. 1 pantallas]. Available from: https://www.who.int/foodsafety/es/
7. González L, Cortés JA. Revisión sistemática de la resistencia antimicrobiana en enterobacterias en aislamientos intrahospitalarios en Colombia. Biomédica [Internet]. 2014;34(2):180-97. https://doi.org/10.7705/ biomedica.v34i2.1550

8. Villalobos AP, Barrero LI, Rivera SM, Ovalle MV, Valera D. Vigilancia de infecciones asociadas a la atención en salud, resistencia bacteriana y consumo de antibióticos en hospitales de alta complejidad, Colombia, 2011. Biomédica [Internet]. 2014;34(Sup1):67-80. https://doi. org/10.7705/biomedica.v34i0.1698

9. Oggioni MR, Dowson CG, Smith JM, Provvedi R, Pozzi G. The tetracycline resistance gene tet(M) exhibits mosaic structure. Plasmid. [Internet]. 1996;35(3):156-63. https://doi. org/10.1006/plas.1996.0018

10. Lavilla Lerma L, Benomar N, Knapp CW, Correa Galeote D, Gálvez A, Abriouel H. Diversity, distribution and quantification of antibiotic resistance genes in goat and lamb slaughterhouse surfaces and meat products. PLoS One. 2014;9(12). https://doi.org/10.1371/journal. pone. 0114252

11. CLSI: Clinical \& Laboratory Standards Institute. [Internet]. St. Louis, Missouri, Estados Unidos; [enero de 2017; citado 30 enero 
2019]. CLSI M100-27. Performance standards for antimicrobial susceptibility testing. [aprox. 1 pantallas]. Available from: https://clsi.org/ media/1469/m100s27_sample.pdf

12. Pennacchia C, Ercolini D, Villani F. Spoilage-related microbiota associated with chilled beef stored in air or vacuum pack. Food Microbiol. 2011;28(1):84-93. https://doi.org/10.1016/j. fm.2010.08.010

13. Säde $E$, Murros A, Björkroth J. Predominant enterobacteria on modified-atmosphere packaged meat and poultry. Food Microbiol. 2013;34(2):252-8. https://doi.org/10.1016/j. fm.2012.10.007

14. Gribble A, Mills J, Brightwell G. The spoilage characteristics of Brochothrix thermosphacta and two psychrotolerant Enterobacteriacae in vacuum packed lamb and the comparison between high and low pH cuts. Meat Sci. 2014;97(1):83-92. https://doi.org/10.1016/j. meatsci.2014.01.006

15. González-Rey C, Siitonen A, Pavlova A, Ciznar I, Svenson SB, Krovacek K. Molecular evidence of Plesiomonas shigelloides as a possible zoonotic agent. Folia Microbiol (Praha). 2011;56(2):178-84. https://doi.org/10.1007/ s12223-011-0032-2
16. Torbeck L, Raccasi D, Guilfoyle DE, Friedman RL, Hussong D. Burkholderia cepacia: This Decision Is Overdue. PDA J Pharm Sci Technol [Internet]. 2011;65(5):535-43. https://doi. org/10.5731/pdajpst.2011.00793

17. López L, Alfonso, Suárez H. Caracterización microbiológica y molecular de Staphylococcus aureus en productos cárnicos comercializados en Cartagena Colombia. Rev costarric salud pública [Internet]. 2016;25(2):819. Available from: https://www.scielo. sa.cr/scielo.php? script =sci_arttext\&pi$d=$ S1409-14292016000200081\&lng=en.

18. Peixoto $R$ de $M$, de França $C A$, de Souza Júnior AF, Veschi JLA, da Costa MM. Etiologia e perfil de sensibilidade antimicrobiana dos isolados bacterianos da mastite em pequenos ruminantes e concordância de técnicas empregadas no diagnóstico. Pesqui Vet Bras. 2010;30(9):735-40. http://dx.doi: 10.1590/ s0100-736X2010000900005

19. Oguttu JW, McCrindle CME, Makita K, Grace D. Investigation of the food value chain of ready-to-eat chicken and the associated risk for staphylococcal food poisoning in Tshwane Metropole, South Africa. Food Control. 2014;45:87-94. https://doi.org/10.1016/j.foodcont.2014.04.026 
20. Grace D. Food safety in low and middle income countries. Int J Environ Res Public Health. 2015;12(9):10490-507. https://doi. org/10.3390/ijerph120910490

21. Zweifel C, Capek M, Stephan R. Microbiological contamination of cattle carcasses at different stages of slaughter in two abattoirs. Meat Sci. 2014;98(2):198-202. https://doi.org/10.1016/j.meatsci.2014.05.029

22. Doulgeraki Al, Ercolini D, Villani F, Nychas GJE. Spoilage microbiota associated to the storage of raw meat in different conditions. Int J Food Microbiol. 2012;157(2):130-41. https://doi. org/10.1016/j.ijfoodmicro.2012.05.020

23. Hessain AM, Al-Arfaj AA, Zakri AM, El-Jakee JK, Al-Zogibi OG, Hemeg HA, et al. Molecular characterization of Escherichia coli 0157: H7 recovered from meat and meat products relevant to human health in Riyadh, Saudi Arabia. Saudi J Biol Sci. 2015;22(6):725-9. https://doi. org/10.1016/j.sjbs.2015.06.009

24. Jindal BAK, Pandya MK, Khan MID. Antimicrobial resistance: A public health challenge. Med J Armed Forces India. 2015;71(2):178-81. https://doi.org/10.1016/j.mjafi.2014.04.011

25. Iwu C, Iweriebor B, Obi L, Basson A, Okoh A. Multidrug-Resistant Salmonella Isolates from Swine in the Eastern Cape Province, South Africa. J Food Prot [Internet]. 2016;79(7):1234-9. https://doi.org/10.4315/0362-028X.JFP-15-224

26. FAO: Organización de las Naciones Unidas para la Alimentación. Expert Consultation on Community-based Veterinary. FAO Animal production and health. [Internet]. 2004;1:1111. Available from: https://www.fao.org/ docrep/016/y5405e/y5405e00.htm

27. INVIMA: Instituto Nacional de Vigilancia de Medicamento. [Internet].Bogotá, Colombia; [julio 6 de 2017; citado 30 enero 2019]. ABECÉ de la inocuidad de alimentos. [aprox. 5 pantaIlas]. Available from: https://www.minsalud. gov.co/sites/rid/Lists/BibliotecaDigital/RIDE/VS/ PP/SNA/abc-inocuidad.pdf

28. Ministerio de Salud y Protección Social. [Internet].Bogotá, Colombia; [julio 25 de 2013; citado 30 enero 2019]. RESOLUCIÓN 2674 DE 2013 [aprox. 10 pantallas]. Available from: https://www.alcaldiabogota.gov.co/sisjur/ normas/Norma1.jsp?i $=54030$

29. Incontec. NTC 1325. Productos carnicos procesados no enlatados. Icontec. 2008

30. Terentjeva, M., Avsejenko, J., Streikiša, M., Utināne, A., Kovaḷenko, K., \& Bērziṇš, A. Prevalence and antimicrobial resistance of Sal- 
monella in meat and meat products in Latvia. Ann Agric Environ Med. 2017; 24(2), 317-321. https://doi.org/10.5604/12321966.1235180

31. Li, L., Ye, L., Zhang, S., \& Meng, H. Isolation and Identification of Aerobic Bacteria Carrying Tetracycline and Sulfonamide Resistance Genes Obtained from a Meat Processing Plant. Journal of Food Science. 2016 81(6), M1480-M1484.https://doi.org/10.1111/17503841.13318

32. Schwaiger K, Huther S, Hölzel C, Kämpf $P$, Bauer J. Prevalence of antibiotic-resistant enterobacteriaceae isolated from chicken and pork meat purchased at the slaughterhouse and at retail in Bavaria, Germany. Int J Food Microbiol. 2012;154(3):206-11. https://doi.org/10.1016/j.ijfoodmicro.2011.12.014

33. Ojer-Usoz E, González D, Vitas Al, Leiva J, García-Jalón I, Febles-Casquero A, et al. Prevalence of extended-spectrum $\beta$-lactamase-producing Enterobacteriaceae in meat products sold in Navarra, Spain. Meat Sci. 2013;93(2):316-21. https://doi.org/10.1016/j.meatsci.2012.09.009

34. Gwida M, Hotzel H, Geue L, Tomaso H. Occurrence of Enterobacteriaceae in Raw Meat and in Human Samples from Egyptian Retail Sellers. Int Sch Res Not [Internet]. 2014;2014:1-6. http://dx.doi.org/10.1155/2014/565671
35. Arriaga RF, Rojas MT, Navarrete J V, Vargas ES, Castillo AG. Presence of class I integrons in Escherichia coli isolated from meat products in Federal Inspection Type (TIF) plants in the Estado de Mexico. Vet Mex. 2013;44(1):23-30.

36. Anaya PAF, Medina LMR, Ugarriza MEO, Gutiérrez LAL. Determinación de Escherichia coli e identificación del serotipo 0157: H7 en carne de cerdo comercializada en los principales supermercados de la ciudad de Cartagena. Rev Lasallista Investig. 2013;10(1):91-100.

37. Rúgeles LC, Bai J, Martínez AJ, Vanegas MC, Gómez-Duarte OG. Molecular characterization of diarrheagenic Escherichia coli strains from stools samples and food products in Colombia. Int J Food Microbiol. 2010;138(3):282-6.https://doi.org/10.1016/j. ijfoodmicro.2010.01.034

38. Herrera A F, Suárez Q W. Isolation and identification of Listeria spp. from fresh fish samples, marketed in Pamplona (Norte de Santander). Rev UDCA Actual Divulg Cient. 2012;15:257-65.

39. Shawish RR, Al-Humam NA. Contamination of beef products with staphylococcal classical enterotoxins in Egypt and Saudi Arabia. GMS Hyg Infect Control. 2016;11:8. https://doi. org/10.3205/dgkh000268 
40. Carrel M, Zhao C, Thapaliya D, Bitterman $P_{1}$ Kates $A E$, Hanson BM, et al. Assessing the potential for raw meat to influence human colonization with Staphylococcus aureus. Sci Rep. 2017;7(1). https://doi.org/10.1038/s41598017-11423-6

41. Das P, Mazumder P. Prevalence of Staphylococcus in raw meat samples in Southern Assam, India. J Agric Vet Sci [Internet]. 2016;9(1):239. doi:10.9790/2380-09122329

42 Osman K, Badr J, Al-Maary KS, Moussa IMI, Hessain AM, Amin Girah ZMS, et al. Prevalence of the antibiotic resistance genes in coagulase-positive-and negative-staphylococcus in chicken meat retailed to consumers. Front Microbiol. 2016;7. https://doi.org/10.3389/ fmicb.2016.01846

43 Moreno KM. Carbapenémicos: tipos y mecanismos de resistencia bacterianos. Rev Medica Costa Rica Y Centroam LXX [Internet]. 2013;608:599-605. Available from: https:// www.binasss.sa.cr/revistas/rmcc/608/art8.pdf

44. Jans C, Sarno E, Collineau L, Meile L, Stärk KDC, Stephan R. Consumer exposure to antimicrobial resistant bacteria from food at Swiss retail level. Front Microbiol. 2018;9. https:// doi.org/10.3389/fmicb.2018.00362
45. Yassin AK, Gong J, Kelly P, Lu G, Guardabassi $L$, Wei $L$, et al. Antimicrobial resistance in clinical Escherichia coli isolates from poultry and livestock, China. PLoS One. 2017;12(9). https:// doi.org/10.1371/journal.pone.0185326

46. Mateus-Vargas RH, Atanassova V, Reich F, Klein G. Antimicrobial susceptibility and genetic characterization of Escherichia coli recovered from frozen game meat. Food Microbiol. 2017;63:164-9. https://doi.org/10.1016/j. fm.2016.11.013

47. Buzón-Durán L, Capita R, Alonso-Calleja C. Microbial loads and antibiotic resistance patterns of Staphylococcus aureus in different types of raw poultry-based meat preparations. Poult Sci. 2017;96(11):4046-52. https://doi. org/10.3382/ps/pex200

48. Kim MS, Lim $T H$, Jang JH, Lee $D H$, Kim BY, Kwon JH, et al. Characteristics of the antimicrobial resistance of Staphylococcus aureus isolated from chicken meat produced by different integrated broiler operations in Korea. Poult Sci. 2018;97(3):962-969. https://doi. org/10.3382/ps/pey335

49. Baylis C, Uyttendaele $M$, Joosten $H$, Davies A, Heinz HJ. The Enterobacteriaceae and their significance to the food industry. ILSI Europe Report Series. 2011:1-48. 
50. Fajardo-Zapata ÁL, Méndez-Casallas FJ, Molina LH. Residuos de fármacos anabolizantes en carnes destinadas al consumo humano. Univ Sci. 2011;16(1):77-91.

51. Aidara-Kane A. Containment of antimicrobial resistance due to use of antimicrobial agents in animals intended for food: WHO perspective. Rev Sci Tech [Internet]. 2012;31(1):27787. Available from: https://doi.org/10.20506/ rst.31.1.2115

\section{(@) $(\Phi \otimes$}

Esta obra está bajo una licencia de Creative Commons Reconocimiento-NoComercial 4.0 Internacional 